\title{
Gray level histogram width tissue characterization
}

\begin{abstract}
Purpose: Proposal to study liver with gray level histogram width (GLHW), clinical ultrasound tissue characterization, using usual B-mode devices.

Materials: Placental intervillous space fibrin deposit in fetal growth restriction (FGR), malignant neoplasia, fetal brain, immature fetal lung, meconium-stained amniotic fluid, and healthy adult liver.

Methods: Commercial ultrasound B-mode histogram base width divided by full gray scale length was gray level histogram width (GLHW), and it was used to tissue characterization.

Results: GLHW of ovarian, cervical and endometrial malignancies were 50 or higher $\%$. Fibrin deposit in placental intervillous space was diagnosed with GLHW and treated with heparin administration to obtain normal neonate. Fetal brain echo density, immature fetal lung, meconium stained amniotic fluid, and healthy adult liver GLHW were studied with GLHW. Helsinki declaration was followed.

Conclusion: The GLHW objectively diagnosed ultrasound B-mode image, thus, it would be applied even in adult human cases.

Keywords: ultrasound, GLHW, tissue characterization, placenta, FGR, malignancy, fetal organ, liver, radiation measurement inc, respiratory distress syndrome, region of interest, periventricular echodensity, ultrasonic b-mode, intervillous fibrin, hydrocortisone, growth factor, erythropoietin
\end{abstract}

Volume 3 Issue I - 2017

\author{
Maeda K,' Kihaile PE,' ITOT,' Utsu M, ${ }^{3}$ \\ Yamamoto $\mathrm{N},{ }^{3}$ Serizawa $\mathrm{M}^{4}$ \\ 'Department of Obstetrics and Gynecology, Hakuai Hospital, \\ Japan \\ ${ }^{2}$ Department of Obstetrics and Gynecology, Seirei-Mikatahara \\ Hospital, Japan \\ ${ }^{3}$ Department of Obstetrics and Gynecology, Hamamatsu \\ Medical Center, Japan \\ ${ }^{4}$ Department of Obstetrics and Gynecology (Emeritus), Tottori \\ University Medical School, Japan
}

\begin{abstract}
Correspondence: Kazuo Maeda, Department of Obstetric and Gynecology, 3-I 25, Nadamachi, Yonago, Tottoriken, 6830835, Japan, Tel 81859226856, Fax 8|859226856,

Emailmaedak@mocha.ocn.ne.jp
\end{abstract}

Received: October 03, 2016 | Published: March 12, 2017
Abbrevations: GLHW, gray-level histogram width; RMI, radiation measurement inc; ROI, region of interest; FGR, fetal growth restriction; PVE, periventricular echodensity; PVL, periventricular leukomalacia; RDS, respiratory distress syndrome

\section{Introduction}

Although ultrasound B-mode image diagnosis was superb, objective tissue echogeninity characterization was desired, however, particular computer and program were mandatory. ${ }^{1}$ Ultrasound B-mode gray-level histogram width (GLHW) achieved clinical tissue characterization in obstetrics and gynecology.

\section{Methods}

The clinical tissue characterization was achieved by gray level histogram width divided by full gray scale length Figure $1^{2,3}$ of which value was called gray level histogram width (GLHW), which was standardized by studying RMI 412 phantom (Radiation Measurement Inc. Middleton, Wisconsin), of which histogram width did not change when the B-mode device gain controled, while image contrast had to be lowest, because histogram width was enlarged when the image contrast was high. The GLHW. of RMI 412 phantom was studied to be standardized in various Aloka machines (Aloka, Tokyo) and Voluson 530D (GE Healthcare)., The GLHW was manually calculated, and also automatically calculated by " $\% \mathrm{~W}$ " index in the histogram of Aloka B-mode devices (Tokyo), where the value was the same as manual determination. ${ }^{3}$

\section{Materials and results}

\section{Diagnosis of malignancy}

GLHW in preoperative ovarian masses were compared to postoperative pathology in benign and malignant tumors, ${ }^{4}$ where mean GLHW was $18 \pm 10 \%$ in benign masses, while it was $51 \pm 11 \%$ in malignancy, and mean GLHW values were larger in malignant masses than benign tumors Figure $2,{ }^{4}$ and therefore, ovarian malignant neoplasia is diagnosed by GLHW. Ovarian dermoid cyst was intermediate between benign and malignant masses; however, a dermoid cyst is diagnosed by its characteristic B-mode image, which is the niveau formation.

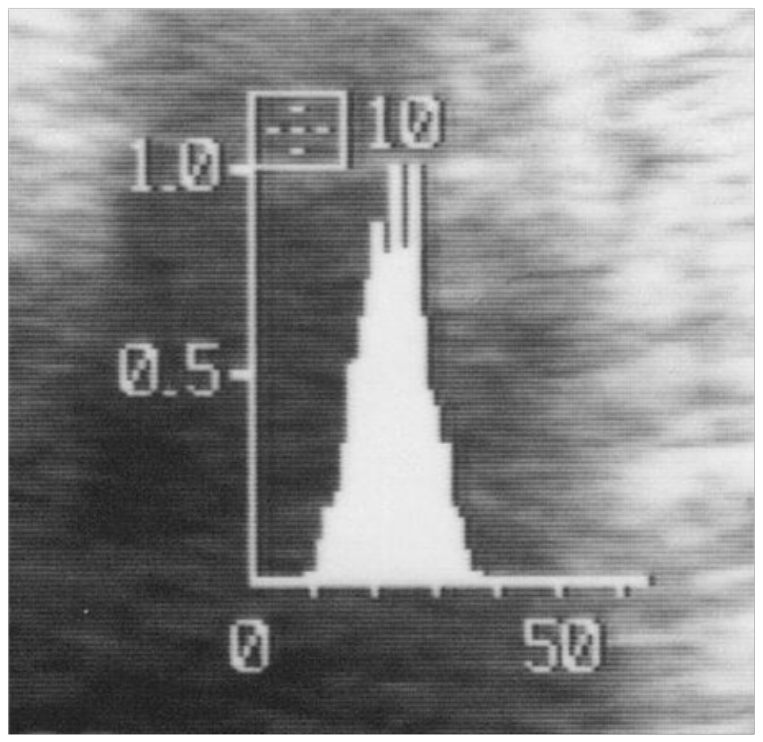

Figure I The calculation of GLHW In the histogram of usual diagnostic ultrasound B-mode device. H, Gray level histogram width. W, full gray scale length. $\mathrm{H} / \mathrm{W}(\%)$ is GLHW, which is automatically obtained by the \%W index of ultrasound histogram of Aloka diagnostic B-mode devices. 


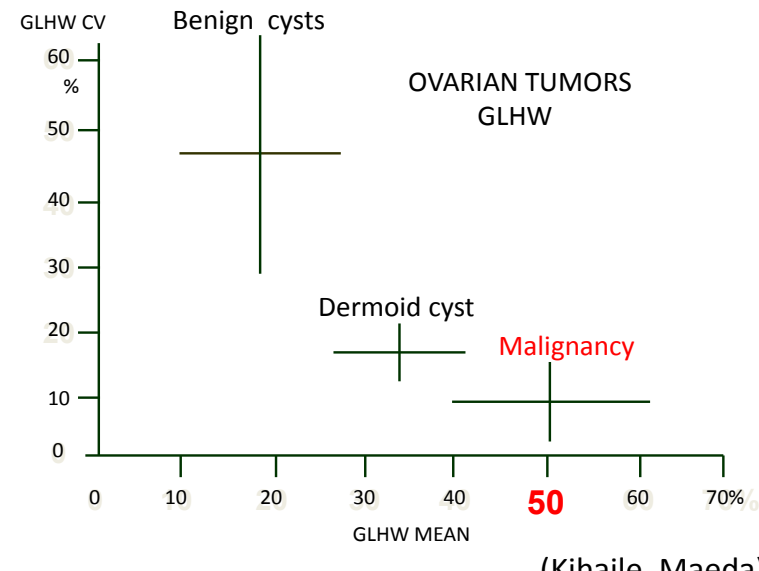

Figure 2 Ovarian tumor GLHW values, which was larger in malignancy than benign one, while coefficient of variation (CV) was larger in benign one than malignancy. There was significant difference between GLHW values of benign and malignant ovarian tumors. ${ }^{4,6}$

In other studies, mean GLHW was $42.7 \pm 5.0 \%$ in normal endometrium, while it was $58.2 \pm 11.2 \%$ in endometrial cancer, thus, endometrial cancer will be diagnosed using GLHW. ${ }^{5,6}$

The author found that the GLHW of a uterine cervical cancer was higher than $50 \%$. Thus, general malignancy will be indicated, if its GLHW is 50 or $\operatorname{more} \%$.

Recently, Nam et al. ${ }^{7}$ reported differentiation of malignant and benign thyroid nodules using histogram analysis of gray scale sonograms. Ultrasonic B-mode histogram diagnosis of malignancy was supported also by the report.

\section{Grade 3 placenta and intervillous fibrin deposit}

GLHW of $1 \mathrm{~cm}^{2}$ region of interest (ROI) of placental image was manually determined in every 2 gestational weeks in 20 to 41 weeks of normal pregnancies, where mean $\pm 1.5 \mathrm{SD}$ of GLHW were determined. The GLHW was larger in grade 3 placenta than normal placenta. ${ }^{3}$

\section{Placental fibrin deposit in FGR treated by heparin}

Placental GLHW of a case of IUGR (fetal growth restriction, FGR) was larger than that of normal placenta and diagnosed by Utsu to be intervillous fibrin deposit (Figure 3) due to positive cardiolipin antigen in the pregnant woman, and daily 5,000 unit heparin was administered to the woman. The GLHW decreased, estimated fetal weight increased to normal, and normal neonate was obtained (Figure $4),{ }^{3}$ despite an FGR fetus died in previous pregnancy of the mother in the present study. ${ }^{3}$ Discussion: The deposited fibrin would reduce placental active transfer of fetal nourishing material developing FGR, and further damaged passive transfer of oxygen would cause fetal demise in previous pregnancy. Heparin solved deposited fibrin to promote placental transfer function then treated FGR and prevented fetal demise after hypoxia.

\section{Fetal brain periventricular echogenesity}

Yamamoto et al. ${ }^{8}$ studied fetal brain in preterm pregnancy detecting periventricular echodensity (PVE) (Figure 5), of which 18\% (corresponding $0.2 \%$ of all births) preceded neonatal periventricular leukomalacia (PVL) followed by cerebral palsy (CP), if the PVE lasted until preterm birth, while there was no neonatal PVL when the PVE disappeared before birth. Also, no PVE developed in fullterm births' neonates. The GLHW of fetal PVE was $36 \pm 5 \%$ which was significantly larger than $23 \pm 5 \%$ of normal fetal brain GLHW, ${ }^{8}$ thus, GLHW is useful to diagnose fetal and neonatal brain PVE in the prevention of neonatal PVL and CP, namely, the PVE ultrasonically detected immediately after a preterm birth will be effectively treated administrating medicines, e.g. growth factor, erythropoietin, hydrocortisone etc, in the future.

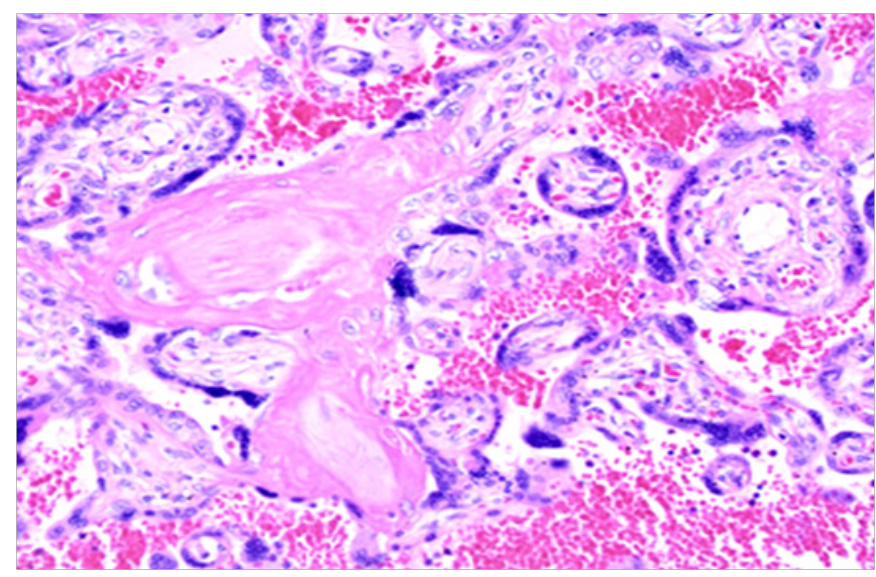

Figure 3 Microscopic view of a placental intervillous space fibrin deposit. $\mathrm{HE}, 200 \mathrm{x}$.

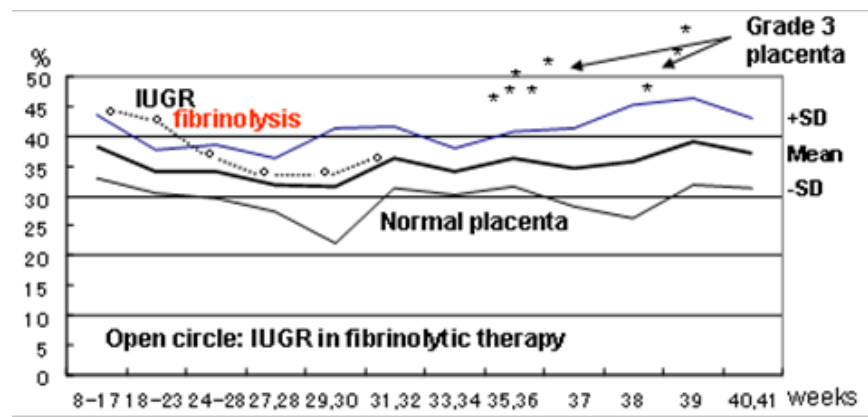

Figure 4 Repeated GLHWs of a FGR (open circles) were compared to normal placental GLHW (Mean \pm I.5SD). High GLHW of the FGR placenta decreased to normal level by the heparin treatment, which soluted fibrin, where estimated fetal weight increased to normal level, then normal neonate was born.

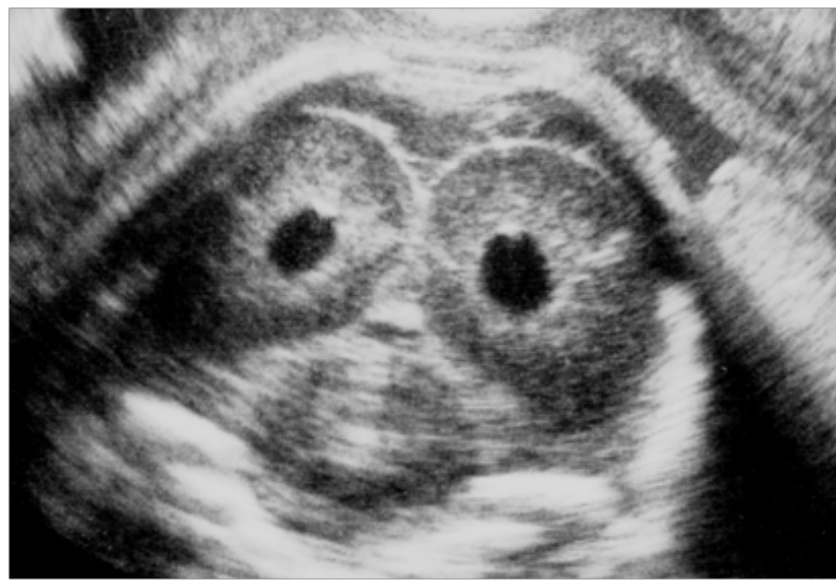

Figure $5 \mathrm{~A}$ case of fetal brain PVE, which was white tissues around fetal ventricles in ultrasound $\mathrm{B}$-mode image. It persisted until preterm birth in the case then changed to periventricular leukomalacia, followed by cerebral palsy. 


\section{Diagnosis of immature fetal lung}

Fetal lung maturity should be detected prior to preterm birth to treat the neonate with artificial surfactant to prevent respiratory distress syndrome (RDS). Since the amniocentesis to analyze amniotic fluid for the detection of fetal lung immaturity is invasive at present perintal medicine, non-invasive ultrasonic tissue characterization of immature fetal lung has been studied by Serizawa. ${ }^{9-11}$

GLHW values of fetal lung declined in immature fetal lung, while fetal liver GLHW was constant, the ratio of fetal lung GLHW and fetal liver GLHW was obtained, where fetal lung was immature in young fetus, when the result of gestational weeks multiplied by the ratio of fetal lung to liver ratio was less than 29 , i.e. it was the critical level to divide immature and mature fetal lung, namely, 96\% of neonatal respiratory distress syndrome (RDS) was predicted by the value lower than 29. The detection rate was highest among various RDS detection parameters. Novel preterm pregnancy management, e.g. maternal steroid administration is monitored by repeated noninvasive GLHW studies. Neonatal surfactant therapy is prepared, or fetal steroid therapy will be repeated, if the fetal lung is still immature with GLHW study. ${ }^{10}$

\section{Diagnosis of meconium stained amniotic fluid}

Amniotic fluid is clear flud accumulated around the fetus in pregnant uterus, which was stained by meconium, fetal stool, was released into amniotic fluid in hypoxic fetal asphyxia. The condition is diagnosed with amnioscopy by Saling in 1950s before fetal heart rate monitoring. As the amniotic fluid was stained yellowish dirty, by meconium, expelled through relaxed fetal anal muscle in asphyxia, amniotic fluid GLHW was higher than clear fluid, and its GLHW was similar to that of fetal colon. Results were confirmed by Yamamoto ${ }^{11}$ where normal fluid GLHW was $6.7 \pm 2.0 \%$, Colon $9.5 \pm 2.3 \%$ and meconium stained fluid $11.0 \pm 3.6 \%$, where significant difference was present between meconium-stained and normal fluid, while the difference was insignificant between colon and stained fluid, and significant between colon and normal fluid. Meconium stained fluid would be diagnosed by ultrasound GLHW, namely, fetal asphyxia will be detected by GLHW of amniotic fluid.

\section{GLHW of healthy adult liver}

Mean GLHW of 33 healthy adult liver was $34.8 \pm 3.7 \%$ and its coefficient of variation was $10.5 \%$, where no relation of GLHW was noted to the age or gender. ${ }^{3}$ Therefore, pathological state of adult liver is preferable to be studied by its specialists using GLHW, which is determined by the division of histogram base width by full gray scale length in the ultrasound B-mode, or simply by the "\%W" parameter in the Aloka ultrasound B-mode histogram.

\section{Conclusion}

Gray level histogram width (GLHW) is update clinical ultrasound tissue characterization calculated from ultrasound B-mode echogenicity histogram parameter in commercial B-mode imaging device. Placental intervillous space fibrin deposit, malignant neoplasia, fetal brain echogenesity, immature fetal lung, meconiumstaind amniotic fluid and normal adult liver were studied, where GLHW was useful non-invasive clinical tissue characterization using commercial B-mode devices.

\section{Acknowledgements}

None.

\section{Conflict of interest}

Author declares that there is no conflict of interest.

\section{References}

1. Akaiwa A. Ultrasonic attenuation character estimated from backscattered radiofrequeny signals in obstetrics and gynecology. Yonago Acta Med. 1989;32(1):1-10.

2. Maeda K, Akaiwa A, Kihaile PE. Ultrasound Tissue Characterization. Ultrasound in Obstetrics and Gynecology Little Braun, Boston, USA; 1993. p. 55-59.

3. Maeda K, Utsu M, Kihaile PE. Quantification of sonographic echogenicity with grey-level histogram width; A clinical tissue characterization. Ultrasound Med Biol. 1998;24(2):225-234.

4. Kihaile PE. Ultrasonic tissue characterization of ovarian tumors by the scanning of grey-level histograms. Yonago Acta Medica. 1989;32(3):251-260.

5. Maeda K, Utsu M, Yamamoto N, et al. Clinical tissue characterization with gray level histogram width in obstetrics and gynecology. The Ultrasound Review of Obstetrics and Gynecology. 2002;2(2):124-128.

6. Ito T. Diagnosis of endometrial cancer with GLHW tissue characterization. Personal communication. 2007.

7. Nam SJ, Yoo J, Lee HS, et al. Quantitative evaluation for differentiating malignant and benign thyroid nodules using histogram analysis of grayscale sonograms. J Ultrasound Med. 2016;35(4):775-782.

8. Yamamoto N, Utsu M, Serizawa M, et al. Neonatal periventricular leukomalacia preceded by fetal periventricular echodensity. Fetal Diagn Ther. 2000;15(4):198-208.

9. Maeda, Serizawa M, Utsu M, et al. Echogenicity of fetal lung and liver quantified by the gray-level histogram width. Ultrasound Med Biol. 1999;25(2):201-208.

10. Serizawa M, Maeda K. Noninvasive fetal lung maturity prediction based on ultrasonic gray level histogram width. Ultrasound Med Biol. 2010;36(12):1998-2003.

11. Maeda K, Serizawa M, Yamamoto N. Ultrasound tissue characterization with the gray level histogram width of the B-mode. The Ultrasound Review of Obstetrics and Gynecology. 2005;5(2):92-95. 